\title{
Exploring the possibilities to increase the autonomy of an electric vehicle
}

\author{
Răzvan Marcuș ${ }^{1}$, Olimpiu Stoicuța $^{1}$, Cosmin Rus $^{1 *}$, and Bogdan Tomus ${ }^{2}$ \\ ${ }^{1}$ University of Petrosani, Department of Automation, Computers, Electrical Engineering and \\ Energetics, University Street 20, Petroșani, 332006, Romania \\ ${ }^{2}$ University of Petrosani, Department of Mechanical, Industrial and Transportation Engineering, \\ University Street 20, Petroșani, 332006, Romania
}

\begin{abstract}
The paper identifies the possible energy sources that an electric vehicle can use during its operation to increase energy autonomy. The identified sources are modeled and simulated, and the results are interpreted in terms of viability. The paper presents the constructive principles of a power regeneration system consumed by an electric vehicle during operation to increase its autonomy. It also identifies the possible energy sources that an electric vehicle can use during its operation. The concepts of electricity regeneration are modeled and simulated, and the results are interpreted in terms of viability.
\end{abstract}

\section{Introduction}

Electric power is the vital element for the operation of electric vehicles and all the equipments it contains. In the existing electric vehicle we rarely have a system that would constantly generate electric power as is the case with cars that have a thermal engine on fuel. Within the propulsion system that is using fossil fuels is attached an alternator that performs the function of an electric generator, which supplies electricity for the functioning of the electronic equipment within the car. In electric vehicles, there is no "alternator" that could produce electric power while moving and accelerating. There are electrical regeneration systems that function during the "motor brake" or electromagnetic braking. With these systems, there is brought only a small vehicle autonomy, it has a fixed power capacity with which it starts on the road and then it is still downloading according to necessities. One of the major difficulties of electric vehicles is the electrical energy storage capacity of the batteries, the cost of the batteries, the dimensions of the batteries and their weight, which impose major problems in the design and construction of electric vehicles. If there is needed a larger amount of energy stored inside the batteries, their volume increases as well as their weight and there all the costs involved get higher [1]. The paper proposes a new system for regenerating part of the electricity consumed by an electric vehicle. It is

Corresponding author: author@email.org 
proposed to create a system consisting of two electricity generators driven by the vehicle's movement, which together with a photovoltaic system controlled by an maximum power point tracking (MPPT) system will ensure greater autonomy as well as increased reliability.

\section{Electric regeneration system applied to an electric vehicle}

The electrical energy regeneration system we have identified as being most useful is the producing of electric power while the vehicle is moving, accelerating or even maintaining constant speed. In order to have as low as possible energy consumption, the engine construction must be one with permanent magnets, brushless model and with hall sensors. Thus, the impulses that are sent to perform the rotation movement are accurate, given at the right moment and with low energy consumption.

To be very efficient for the power regeneration part and consequently to increase autonomy, the system have to work in parallel with the vehicle, so it will generate electricity at all running speeds [2].

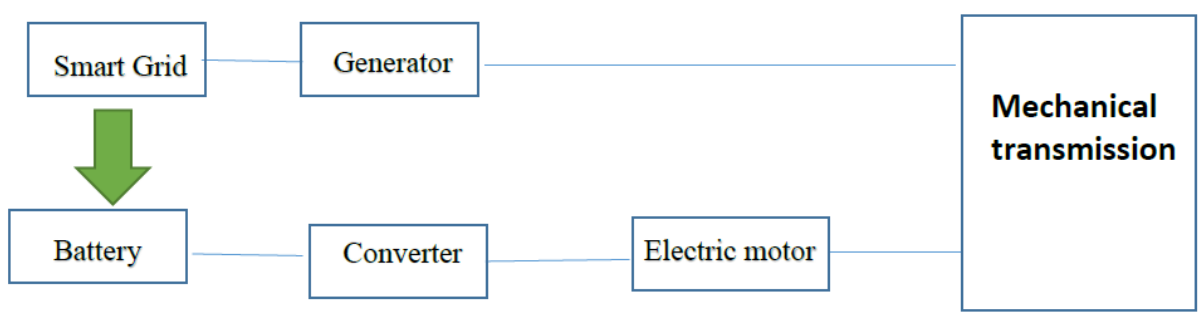

Fig.1. Block diagram of an electric vehicle

According to the above diagram, the operating system is parallel and the novelty is the first branch with the generator and the smart grid. The generator is one with permanent magnets without torque, so the friction is only on the supporting bearings, there is no other physical contact and resistance to lock them. The generator is directly connected to the mechanical transmission and has a fixed gear ratio that is directly proportional to the speed of the vehicle. Because there are vehicle speed variations from $0 \mathrm{~km} / \mathrm{h}$ to $130 \mathrm{~km} / \mathrm{h}$ and battery voltage is 56 volts, a smart system is required to achieve battery charging and takeoff voltage fluctuations on the generator [3].

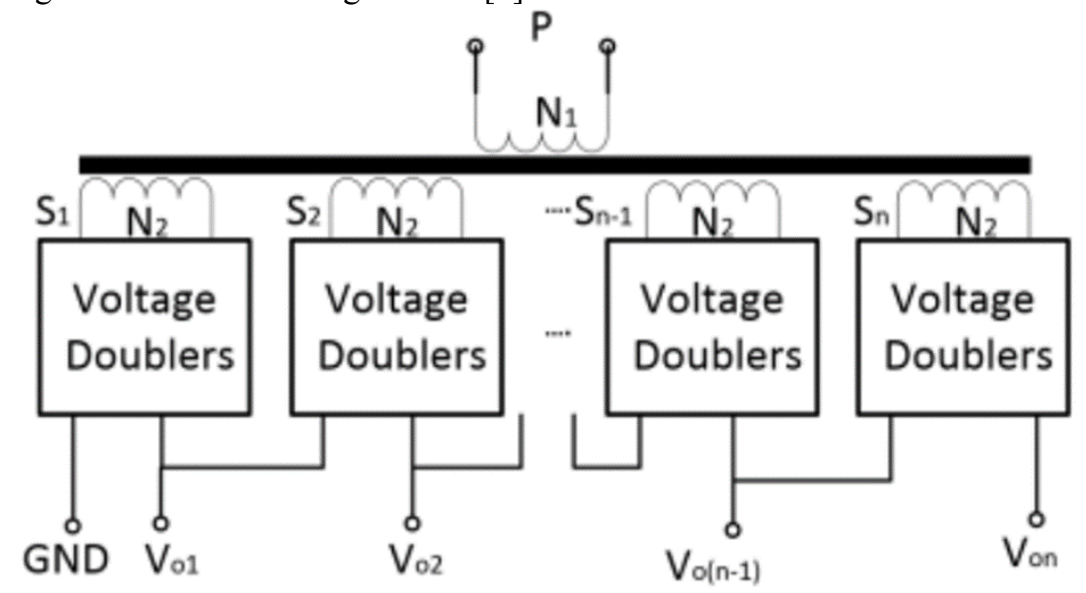

Fig. 2. Electric transformation system 
As it is shown in the figure above (figure 2), the transformation system will be made with the possibility of several output stages depending on the desired voltage. In order to be able to select the optimal voltage for the operation of the battery charging and maintenance system, the operation is done in stages. First of all, the quality of the vehicle's style of handling, the speed of movement and the power absorbed by the vehicle are carefully monitored. During this time, all voltages are monitored on all phases at the output of the generator and on each phase separately, and then one phase is selected which has a voltage range necessary for the operation of the maximum power point tracking system (MPPT) for charging the batteries. Voltages measurement is achieved with the help of voltage dividers, as we work with very high voltages and the controllers can handle only low voltages [2].

The generator, at a running speed of $10 \mathrm{~km} / \mathrm{h}$, produces a voltage of about 500 volts $\mathrm{AC}$, but to charge the batteries it is required 56 volts, it passes through the transformer and stepped down to a voltage of 100 Volts AC, after stabilization it reaches 120 volts DC but with a gain of current after lowering the voltage. If at the transformer's input we had 500 watts at 500 Watts, the output will have also 500 Watts, but at a voltage of 120 volts DC. After setting the continuous voltage, the $500 \mathrm{~W}$ is introduced and redirected to the common point, which is the connection between the batteries and the electric motor drive system [3]. If the car consumes $50 \mathrm{amps}$ at 56 volts to travel at $10 \mathrm{Km} / \mathrm{h}$, through the regeneration system, which we defraud only with $1 \mathrm{~A}$ but at 500 volts, we can save about 10 amperes in the system from the car's consumption and so we extend the travel distance by $21.95 \%$. With this performance, we can lower the car's weight, using fewer batteries and having lower costs. When driving the vehicle at $80 \mathrm{~km} / \mathrm{h}$, the generator system produces 15000 volts $\mathrm{AC}$, this is a very high voltage, but with the winding selection on the transformer we can redress the voltage to a lower one, from there we lower it with the transformer at 100 volts $\mathrm{AC}$, and through stabilization and conversion we bring it to 120 volts DC. From these high voltages, we need very small currents to regenerate enough power to move the vehicle. For a consumption of only 1 A per generator at 15000 volts, we have an available power of about $15 \mathrm{~kW}$ at a speed of $80 \mathrm{~km} / \mathrm{h}$. These values are theoretical and ideal, but in practice there are losses through the transfer part, by the release of the temperature and the different quality of the conductive material. To reduce emissions and increase efficiency, the system is put in a special enclosure that is naturally ventilated by air currents, all to keep the electromagnetic field closed [4].

\section{Battery charging system}

The charging system using MPPT is one of the most efficient with the right algorithms. This type of system comes from photovoltaic systems that take power from the input and convert the same power to output but to other voltages and currents. There are other forms of battery charging from pulsed photovoltaic systems, the system called PWM, but it is not as efficient, the input power is not equal to the power output at a time. Maximum power point tracking (MPPT) is an independent algorithm and most commonly used in photovoltaic systems to continuously adjust the impedance seen at the solar panel to maintain the photovoltaic system operating at or near the top of the panel under different conditions such as scratching solar irradiation, temperature and charge. The MPPT algorithms are designed to maximize the power generated by our system, which continuously monitors the input voltage to ensure that the system operates at maximum power on the supply voltage curve. For photovoltaic systems, the algorithms are used for cumulative factors such as radiation and temperature, but in this case the load variation is needed compared to the speed of movement, the increase in the degree of acceleration and the degree of discharge of the batteries. In figure 3 we have an algorithm for disturbing and 
observing the supply voltage to provide maximum power. This is the most used charging system but it is not the most advanced system [5].

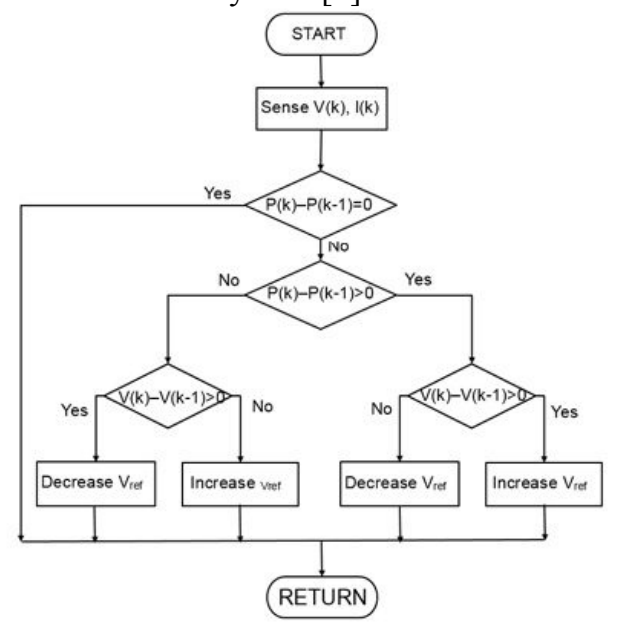

Fig.3. Algorithm for disturbing and observing the supply voltage used in MPPT systems

Figure 4 shows a more advanced control system for MPPT. It compares incremental strength with instantaneous resistance in a system. Depending on the result, it increases or decreases the voltage until reaching the maximum power point. Unlike the previous system, the voltage remains costly once the maximum value is reached [5].

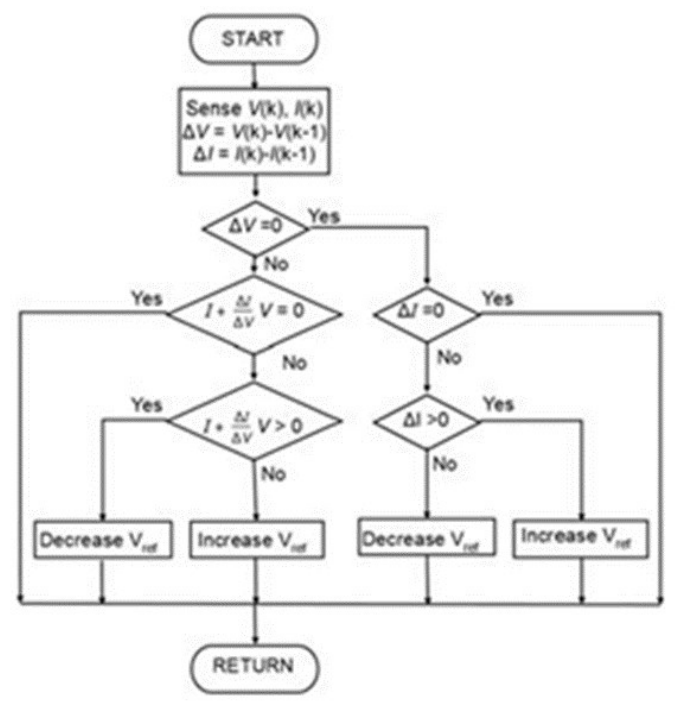

Fig.4. Advanced control system for MPPT

The take-up and use of smart charging and management systems is the creation of a favorable energy gain ratio. The use of the MPPT charging system in our case is most advantageous as we have a power transfer from the generator to the minimal losses but also with minimal deflections. The generator can be mounted even on the direct drive motor axis or on any other motor axis that will be able to take over the indirect rotation movement to be transferred to the generator by rigid transmission [6]. 


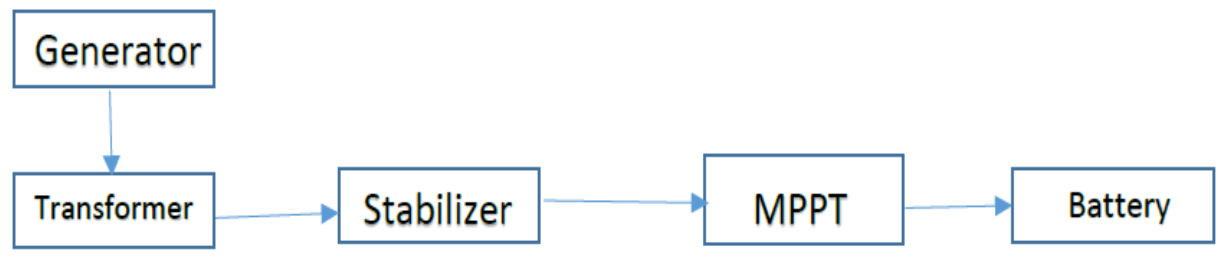

Fig.5. Scheme of integration of MPPT into the charging system

According to the above scheme, the use of the MPPT system in our work will control the energy absorption of the transformer by reading several parameters, namely:

- the degree of acceleration of the vehicle

- battery charge level

- speed of the vehicle

- the amount of energy absorbed by the batteries

- battery temperature

- generator speed

- phase measurement on the transformer

After all these parameters have been monitored, the charging system will have a charging start delay until a small amount of battery power stabilization is read [7]. Once it has established a constant power consumption on the batteries, it will gradually increase the amount of energy it will supply to the battery or motor. In this sense he will see the amount of energy that he will supply to the system and the maximum amount of current that he will be defending [8]. In this paper we aim to gradually increase the load so as to cover a percentage of the energy consumption of the car for its movement and thus not to defeat more than $3 \mathrm{~A}$ at the voltage of $48 \mathrm{~V}$. At a consumption of 1 ampere and $200 \mathrm{~V}$ when is powered MPPT, it will be able to supply $3 \mathrm{amp}$ to 48 volts to charge the car, so the gain of energy that will be achieved by excess power on the engine will be reintroduced into its system [8]. But for daytime battery storage at a constant voltage, small photovoltaic panels will be used to cover all surfaces that are exposed to light in order to maintain the entire energy system of the car and also with the second MPPT system which will monitor the insulating resistance of the panels will keep the batteries cool so they do not deteriorate or there is a risk of their sulphation. This MPPT is able to carry out several loading methods in various stages plus controlled battery discharge, which allows them a much longer life [9, $10]$. According to figure 6 , there are these 2 battery maintenance systems, the photovoltaic system comes with extra electricity even at the time of stationing, it exerts on the accumulators all the loading stages with a constant load in the capacity of the photovoltaic panels, short accumulations for decomposing plate deposits and eliminating sulphation [8].

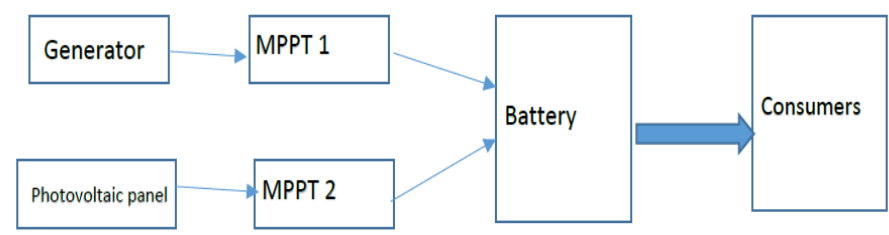

Fig.6. Charging system with two MPPTs 
The whole system works in parallel, the two MPPTs work together with each other and coordinate all of its functions, so in MPPT 2, it will not put the batteries in short to make their downloads when it works, it will only take power up to accumulators or directly to consumers.

\section{Conclusion}

This paper presents an innovative method of recovering a part of the electric energy consumed by an electric vehicle. The system consisting of 2 generators, one dynamic and the second stationary will increase the efficiency and distances traveled by the car, up to $45 \%$ more than when these systems are not installed. The use of the photovoltaic system with one MPPT, especially when the vehicle is stationary for a long time, keeps the batteries alive and recharges at maximum capacity without energy consumption from the national grid. Applications of photovoltaic systems are increasingly present in the area of interest of electric vehicles, especially since the new technologies allow the creation of photovoltaic panels that bend according to the shape of a vehicle. This application is not the only feasible in this field of activity, it can be found a multitude of practical applications where it can be apply these principles, among which the most important would be the applications that have as main area of interest the development of complex energy storage systems. systems in order to increase the autonomy of an electric system. These battery management systems find their best applicability in the field of electric vehicles by the simple fact that they increase the autonomy of these vehicles but also increase their reliability by having protection systems that prevent a battery from overcharging or discharging over the allowed limit and possible human errors in the manipulation of the whole system. The system for recovering part of the electricity consumed by an electric vehicle together with a photovoltaic system will greatly increase the autonomy of this type of vehicle and will also reduce the costs arising from the use of heavy batteries that can predispose the entire system to possible defects or accidents.

\section{References}

1. F. Un-Noor, P. Sanjeevikumar, L. Mihet-Popa, M. Nurunnabi, E. Hossain, Energies, 10, 8, (2017)

2. J. M. E. Gomez, A. J. Barragan Pina, E. D. Aranda, J. M. Andujar-Marquez, Appl. Sci., 8, $19,(2018)$

3. M.R. Banaei, A.F. Bonab Hossein, International Journal of Circuit Theory and Applications, 45, 8, (2017)

4. T.J. Liang, J.H. Lee, IEEE Transactions on Power Electronics, 31, 6, pp. 4324-4330, (2016)

5. E.H. Ismail, A.A. Fardoun, A.A. Zerai, Int. J. Circ. Theor. Appl., 42, pp. 358-375, (2014)

6. H. Qiao, Y. Zhang, Y Yao, L. Wei, Analysis of buck-boost converters for fuel cell electric vehicles, Proc. IEEE Int. Conf. Vehicle Electronic Safety, pp.109-113, (2006)

7. N. Patrascoiu, C. Rus, Quality Access to Success, 20, pp. 347-353, (2019)

8. D.A. Ciupageanu, G. Lazaroiu, International Symposium on Fundamentals of Electrical Engineering (ISFEE), pp. 1-5, (2018)

9. S. R Rosca, M. Leba, MATEC Web of Conferences, EDP Sciences, 121, p. 08006, (2017)

10. M. Leba, S. Riurean, A. Ionica, 2017 12th Iberian Conference on Information Systems and Technologies (CISTI), pp. 1-6, (2017) 